\title{
AS CAUSAS DA OBESIDADE: UMA ANÁLISE SOB A PERSPECTIVA MATERIALISTA HISTÓRICA
}

Aline Fabiane Barbieri, Universidade Estadual de Maringá, Maringá, Rio Grande do Sul - Brasil

Rosângela Aparecida Mello, Universidade Estadual de Maringá, Maringá, Rio Grande do Sul Brasil

\begin{abstract}
RESUMO
A obesidade é um grave problema de saúde pública na sociedade atual. Inúmeras são as pesquisas científicas direcionadas ao estudo das causas do desenvolvimento da obesidade. Em contrapartida, a prevalência de obesidade vem aumentando exponencialmente, atingindo pessoas de todas as faixas etárias. Em busca da compreensão aprofundada e radical das causas da obesidade, fizemos uma análise desse tema a partir do pressuposto materialista-histórico de Karl Marx e Friedrich Engels. Nesse sentido, nessa pesquisa objetivamos, sobretudo, compreender o fundamento histórico-social do desenvolvimento da obesidade e seus determinantes nesse momento histórico. Como conclusão, identificamos que os fatores causadores de obesidade estão enraizados na estrutura e funcionamento da sociedade capitalista, sistema de organização social onde o lucro é o objetivo final do trabalho humano.
\end{abstract}

Palavras-Chave: Obesidade-Causas; Saúde; Materialismo-Histórico.

\section{THE CAUSES OF OBESITY: AN ANALYSIS FROM A HISTORICAL PERSPECTIVE MATERIALISTIC}

\begin{abstract}
Obesity is a serious public health problem in society today. There are numerous scientific researches directed to the study of the causes of obesity. In contrast, the prevalence of obesity has increased exponentially, reaching people of all ages. In search of deeper understanding and radical causes of obesity, we analyzed this issue from the historical-materialist assumption of Karl Marx and Friedrich Engels. Accordingly, this study aimed mainly to understand the historical background and social development of obesity and its determinants in this historic moment. In conclusion, we identified the factors that cause obesity are rooted in the structure and functioning of capitalist society, system of social organization where profit isultimate goal of human labor.
\end{abstract}

Key-Words: Obesity-Causes; Health; Historical-Materialism. 


\section{INTRODUÇÃO}

A obesidade está presente em nossa sociedade desde a pré-história, simbolizando, por vezes, beleza e fertilidade. No Período Neolítico as "deusas" eram cultuadas e admiradas por seios, quadris e coxas volumosas. Hipócrates, na medicina greco-romana, já alertava, no entanto, sobre os malefícios da obesidade para a saúde humana ao afirmar que a morte súbita era mais comum em indivíduos com excesso de gordura corporal. No Império Romano, o corpo belo era o corpo magro e esbelto, o que obrigava as damas a fazerem prolongados jejuns. Essa mudança no padrão de beleza, também é possível ser verificada por meio da História da Arte, uma vez que, as obras do século XIII a XX retratam poucas figuras mitológicas ou pessoas obesas. ${ }^{1}$

Ao longo do tempo, por conta do surgimento de novas necessidades humanas, os padrões de beleza e a composição corporal dos homens foram se modificando.

Nas últimas décadas, a prevalência de obesidade vem aumentando exponencialmente, atingindo homens e mulheres de todas as faixas etárias, já sendo caracterizada como uma epidemia mundial. ${ }^{2,3,4}$

De acordo com a Organização Mundial de Saúde (OMS), a obesidade pode ser conceituada como o acúmulo anormal ou excessivo de gordura no organismo que pode levar a um comprometimento da saúde. ${ }^{2}$ Isso porque essa condição corporal pode promover o desenvolvimento de diversas doenças no ser humano, dentre elas, podemos destacar: diabetes mellitus do tipo II e disfunções cardiovasculares, que são, atualmente, as principais causas de morte no Brasil. Além disso, o sujeito obeso tem alta probabilidade de desenvolver vários distúrbios de ordem psico-social, tais como: depressão, transtornos de ansiedade e alteração de imagem corporal. Todas essas consequências, atribuídas e associadas ao excesso de gordura corporal, fazem com que a obesidade, na sociedade contemporânea, seja considerado um grave problema de saúde pública. . $, 3,4,5,6^{2}$

Existem inúmeras pesquisas científicas direcionadas ao estudo das causas do desenvolvimento da obesidade. Tal realidade pode indicar uma possível imprecisão no que diz respeito à identificação das reais causas dessa doença.

Tendo em vista a necessidade de caminharmos em direção ao conhecimento das causas da obesidade, nessa pesquisa, optamos como matriz teórica o materialismo-histórico. 
A concepção materialista-histórica instaura uma compreensão inteiramente diferente das outras correntes teórico-filosóficas, uma vez que, explicita a raiz mais profunda do ser social e de sua realidade. É uma concepção que está em sintonia com os interesses históricos da classe proletária, porque, ao permitir a compreensão do processo histórico-social como totalidade, fundamenta uma concepção revolucionária de sociedade. ${ }^{7}$

Essa concepção de história tem como categoria fundamental a totalidade. Sendo assim, não podemos entender um objeto isoladamente. A realidade social é uma totalidade e nenhuma de suas partes pode ser compreendida sem que seja feita as relações com os outros momentos da realidade social.

A premissa básica da concepção materialista-histórica é que não é a consciência que determina a vida, mas a vida que determina a consciência. O ponto de partida para compreender a história, então, deve ser o concreto, o real, o que é objetivo. A história humana é construída, de acordo com essa perspectiva, por meio do trabalho, atividade com a qual os homens se criam e criam toda a realidade social. ${ }^{7}$ Assim, tudo se desenvolve a partir das relações sociais estabelecidas entre os homens, de sua realidade social concreta:

\begin{abstract}
A causa não está na consciência, mas na vida; a causa não está na evolução e na conduta empírica do indivíduo que, por sua vez, dependem das condições universais. Se as circunstâncias em que este indivíduo evoluiu só lhe permitem um desenvolvimento unilateral, de uma qualidade em detrimento das outras, este indivíduo só conseguirá alcançar um desenvolvimento unilateral e mutilado. ${ }^{8}$
\end{abstract}

Podemos entender com isso, que o mesmo acontece em relação ao desenvolvimento das doenças: se as circunstâncias em que o indivíduo evoluiu estimula e potencializa o desenvolvimento de doenças, sua saúde, provavelmente, padecerá.

Feita essa breve introdução a respeito da obesidade e da matriz teórica deste trabalho, podemos entender que buscar a compreensão da obesidade a partir do pressuposto materialista-histórico significa buscar analisar os determinantes sociais da obesidade, ou seja, compreender essa doença na sua articulação com as relações sociais estabelecidas entre os homens, suas condições sóciohistóricas, materiais e objetivas no nosso modo de organização social atual, o capitalismo.

No que se refere aos procedimentos metodológicos da pesquisa, partimos do entendimento de que a corrente teórica do marxismo não apresenta um método, e sim, uma ontologia, ou seja, um estudo do ser social. Sendo assim, não existe um modelo pronto para a realização de pesquisas nessa linha Conexões: revista da Faculdade de Educação Física da UNICAMP, Campinas, v. 10, n. 1, p. 121-141, jan./abr. 2012. 
teórica. Nessa abordagem, parte-se da aparência do objeto, faz-se o processo de abstração, onde se analisa o maior número possível de fenômenos que exerce alguma interferência nesse objeto, e, feito isso, chega-se ao concreto. Porém, como o concreto está sempre em movimento, o que se tem é uma aproximação do que seja o objeto. ${ }^{9}$

Com perspectiva à realização desse movimento, na seguinte pesquisa, buscamos, primeiramente, identificar a realidade, o que significa que buscamos entender a obesidade em nossa sociedade atual, como vem sendo tratada e compreendida, no que diz respeito às suas causas.

Num segundo momento, analisamos os principais determinantes da obesidade apontados pelos artigos científicos à luz de dados científicos, com vistas a apresentar uma forma de entendimento compatível com a realidade social concreta de nosso país.

É importante destacar, sobretudo, que nossa intenção é apenas iniciar uma discussão sobre o assunto. Discussão essa, que acreditamos ser proveitosa para a construção de novos conhecimentos e reflexões sobre a obesidade e demais temas relacionados à saúde na sociedade capitalista.

\section{BREVES CONSIDERAÇÕES SOBRE A DINÂMICA DA SOCIEDADE CAPITALISTA E O DESENVOLVIMENTO DE DOENÇAS}

Para a discussão das causas da obesidade na sociedade capitalista, entendemos como fundamental, primeiramente, tratarmos do funcionamento dessa sociedade.

A sociedade capitalista é formada por classes intermediárias e duas classes sociais fundamentais e antagônicas que a caracterizam: a classe burguesa, composta pelos donos dos meios de produção, e a classe proletária, composta pelos trabalhadores assalariados.

Com o estabelecimento da propriedade privada, temos a divisão social do trabalho e, como consequência, o surgimento da sociedade de classes, do Estado, da alienação da atividade social e, ainda, a repartição desigual, tanto quantitativa quanto qualitativa, do trabalho e dos seus produtos.

E, de acordo com Netto e Braz, ${ }^{10}$ essa repartição desigual é geradora de todas as desigualdades sociais. Isso significa que uma classe terá suas necessidades satisfeitas e a outra, não; uma classe poderá se alimentar adequadamente, com alimentos ricos nutricionalmente, enquanto a outra se 
manterá com os alimentos que puder comprar. Da mesma forma, no que se refere à saúde, uma classe terá maior atenção em saúde e a outra, não.

Para ilustrar essa realidade, podemos utilizar os dados do Instituto Brasileiro de Geografia e Estatística, ${ }^{11}$ sobre os gastos das famílias brasileiras com remédios, de acordo com a renda mensal destas. Nesse sentido, temos que a despesa monetária mensal familiar com remédios de uma família com renda de até $\mathrm{R} \$ 1.245,00$ é de 4,4\% desta quantia, já para as famílias com renda mensal até $\mathrm{R}$ \$ 10.375,00 é de 1,19\%. Estes dados podem estar relacionados aos gastos deste último grupo com planos e seguros de saúde. Enquanto as famílias que tem renda de até R \$ 1.245,00 gastam 0,3\% desta renda com estes serviços mensalmente, as famílias com renda até $\mathrm{R}$ \$ 10.375,00 gastam 2,3\%.

Além disso, não podemos nos esquecer de que a tendência desta sociedade é que tudo se transforme em mercadoria e a indústria farmacêutica lucra com as doenças e não com a cura delas.

De acordo com Lefèvre ${ }^{12}$ a saúde está sempre associada a bens de consumo que objetivam promover a própria saúde. Os medicamentos, os seguros de saúde, os alimentos especiais e os exercícios físicos etc. Para o autor, a saúde é um produto à venda no mercado, através de suas mercadorias específicas.

Isso porque se sabe que no funcionamento da sociedade capitalista, o lucro tem papel central. Ele é o sentido específico da ação do capitalista: a partir de dinheiro, produzir mercadorias para conseguir mais dinheiro e produzir mais mercadorias. ${ }^{10}$

Como o lucro é a força motriz do modo de produção capitalista, esta forma de organização social só pode existir e reproduzir-se na escala em que a busca do lucro é interminável. Capitalistas e empresas capitalistas só existem, e só podem existir, se tiverem no lucro a sua razão de ser, um capitalista e uma empresa capitalista que não se empenharem prioritária e sistematicamente na obtenção de lucros serão liquidados. ${ }^{10}$

Tendo em vista que o objetivo central da sociedade capitalista é o lucro com vistas à manutenção da propriedade privada e divisão de classes, fica claro que o interesse na promoção de saúde do ser humano na sociedade capitalista não está senão voltado à manutenção da força de trabalho (quanto isto corrobora no aumento da produtividade), e, assim, voltado à manutenção da sociedade de classes.

Conexões: revista da Faculdade de Educação Física da UNICAMP, Campinas, v. 10, n. 1, p. 121-141, jan./abr. 2012. 


\section{AS CAUSAS DA OBESIDADE: UMA REVISÃO DE LITERATURA}

$\mathrm{Na}$ busca de identificar o que a literatura científica vem apontando acerca das causas da obesidade, fizemos um levantamento de artigos científicos referentes ao tema, indexados pelo Google Acadêmico e Scielo, publicados no período de 2005 a 2010, tendo em vista que estes indexadores são bastante utilizados para a pesquisa de artigos científicos referentes à obesidade.

Para a pesquisa dos artigos científicos foram utilizadas as seguintes palavras-chave: etiologia, obesidade, causas, causas sociais, saúde pública, saúde coletiva; sendo essas palavras utilizadas tanto isoladas como combinadas. Foram analisados artigos resultantes de revisão de literatura e de pesquisas originais.

Os principais fatores causadores da obesidade apontados pela revisão de artigos científicos estão reunidos no quadro a seguir:

Quadro 1: Os fatores causadores de obesidade apontados pela revisão de literatura

\begin{tabular}{lr}
\hline \multicolumn{1}{c}{ Fatores causadores de obesidade } & $\mathbf{7 5}$ artigos \\
\hline Sedentarismo e alimentação inadequada & $82,66 \%$ \\
Fatores genéticos & $30,6 \%$ \\
Nível socioeconômico & $30,6 \%$ \\
Fatores psicológicos & $21,3 \%$ \\
Fatores demográficos & $16 \%$ \\
Nível de escolaridade & $5 \%$ \\
Desmame precoce & $5 \%$ \\
Ter pais obesos & $3 \%$ \\
Estresse & $2 \%$ \\
Fumo/Álcool & $1 \%$ \\
\hline
\end{tabular}

De uma forma geral, os autores que tratam da etiologia da obesidade destacam seu caráter multifatorial, apontando a obesidade como resultante de vários fatores, atuantes, na maioria dos casos, de forma combinada.

Gonçalves e colaboradores ${ }^{13}$ destacam que esse conceito, multifatorial, aparentemente neutro e já cristalizado na literatura científica, formulado por técnicos de nações de economia central e apontado pela maioria dos artigos que tratam da obesidade, "foi acumulando certa cortina de fumaça sobre os determinantes sociais da doença", apresentando-a como um problema "natural" na sociedade. 
Com vistas a ultrapassarmos essa "cortina de fumaça", a seguir, os fatores causadores da obesidade, apontados pela revisão de literatura, serão abordados particularmente com o intuito de identificarmos os possíveis determinantes destes.

\section{SOBRE O SEDENTARISMO E ALIMENTAÇÃO INADEQUADA COMO FATORES CAUSADORES DA OBESIDADE}

Fisiologicamente, a obesidade é uma condição corporal caracterizada pelo excesso de tecido adiposo no organismo. Já é de consenso na literatura que a obesidade trata-se de uma doença resultante de um desequilíbrio nutricional provocado por um balanço energético positivo que se dá, por sua vez, na medida em que o sujeito ingere mais energia do que é capaz de gastar. Assim, temse um acúmulo de energia que, por ação do hormônio insulina, é convertida a gordura. ${ }^{5,14}$

Tendo em vista esta dinâmica fisiológica, a literatura científica aponta que o desenvolvimento de obesidade está fortemente associado ao "estilo de vida", mais especificamente, no que se refere à prática de atividade física e alimentação. Nesse sentido, sabe-se que quanto mais ativo é o "estilo de vida" de uma pessoa, menor é a probabilidade desta se tornar um sujeito obeso. E quanto mais rica é a alimentação de um sujeito em açúcares, lipídeos e alimentos industrializados, também são maiores as chances deste sujeito tornar-se obeso. $3,6,15,16,17,18,19,20,21,22,23,24,25,26,27,28,29,30,31,32,33$

Vale fazermos aqui, alguns comentários a respeito do termo "estilo de vida", tão utilizado por autores que tratam da obesidade. Dentre os significados da palavra "estilo", encontrada nos dicionários de língua portuguesa, destaca-se: "maneira de exprimir os pensamentos e comportamentos, falando ou escrevendo". ${ }^{34}$ Isso indica que o termo "estilo de vida" se refere à maneira de viver das pessoas, remetendo-nos a uma questão de comportamento individual, de escolhas do sujeito; como se as pessoas pudessem escolher livremente sua maneira de viver: os alimentos componentes de sua dieta, os horários e locais para a prática de exercício físico, sua moradia etc. Certamente essas escolhas são possíveis às pessoas, desde que, no entanto, estas estejam providas de recursos financeiros. Nesse sentido, entendemos que a classe trabalhadora não pode ter um "estilo de vida" em consonância com os seus desejos, uma vez que as condições materiais impostas por essa sociedade impossibilitam isso.

De acordo com Cavalcanti, Dias e Costa, ${ }^{35}$ o aumento da obesidade está tradicionalmente associado com a fartura de alimentos, a mudanças na composição dietética da população ocidental e ao acesso barateado às farinhas e gorduras. A esse respeito, é importante considerar que, com vistas a Conexões: revista da Faculdade de Educação Física da UNICAMP, Campinas, v. 10, n. 1, p. 121-141, jan./abr. 2012. ISSN: 1983-9030 
aumentar a lucratividade, as empresas que produzem alimentos industrializados vêm fazendo combinações de gordura, açúcar e sal que são "hiper-palatáveis". Na verdade, os produtores tentam encontrar um ponto em que as pessoas comam certo produto e fiquem com vontade de comê-lo mais vezes. Assim, salgadinhos, doces e refeições prontas podem ter o mesmo efeito sobre o cérebro que o tabaco. ${ }^{36} \mathrm{E}$, com isso, a ingestão de nutrientes inadequados à saúde se torna cada vez mais frequente.

No que se refere à obesidade infantil, autores destacam que ela está fortemente associada ao desmame precoce e a introdução inadequada de alimentos. ${ }^{6,37}$ Faz-se necessário, no entanto, considerar que a excessiva jornada de trabalho das mães e o tempo gasto no transporte até o local de trabalho podem contribuir para o desmame precoce das crianças e, conseqüente, introdução inadequada de alimentos na dieta. Outro aspecto relacionado por autores da literatura científica é a falta de percepção parental a respeito do estado de obesidade da criança. ${ }^{14}$ Além destes fatores, ainda são apontados: o peso pré-gestacional materno, o fumo durante a gestação e o estado nutricional na infância. ${ }^{3}$ Sobre a obesidade no público adolescente, esses autores afirmam que isso se deve ao consumo de lanches em excesso mal-balanceados e à enorme suscetibilidade desta população à propaganda consumista. De acordo com Rodrigues e Boog, ${ }^{38}$ estamos numa sociedade obesogênica, uma vez que esta nos estimula ao consumo alimentar.

No que se refere à prática de atividade física, sabe-se que a hipoatividade física está diretamente associada com o desenvolvimento de obesidade. ${ }^{20,21}$

Segundo Enes e Slater, ${ }^{3}$ o fato é que houve, ao longo do tempo, uma redução progressiva da prática de atividade física combinada ao maior tempo dedicado às atividades de baixa intensidade, como assistir televisão, usar computador e jogar videogame. Nessa linha de pensamento, também podemos citar Camilo e colaboradores. ${ }^{32}$ Esses autores salientam a influência da evolução tecnológica no estabelecimento de um "estilo" predominantemente inativo da população (classe trabalhadora).

Além do "estilo de vida hipoativo", Lopes, Prado e Colombo, ${ }^{37}$ destacam que, nos últimos anos houve uma tendência da população de um modo geral, em substituir as principais refeições por lanches rápidos, conhecidos como fast-food. Os pais devido ao trabalho e as transformações provocadas pela vida moderna transferem esses hábitos ditos incorretos para seus filhos, prejudicando assim a alimentação adequada das crianças. Ainda de acordo com os autores, a Conexões: revista da Faculdade de Educação Física da UNICAMP, Campinas, v. 10, n. 1, p. 121-141, jan./abr. 2012. ISSN: 1983-9030 
educação dos pais é um fator importante nos hábitos alimentares das crianças. O horário de se realizar as principais refeições bem como a seleção dos alimentos consumidos é passado de pais para filhos. ${ }^{37}$

A questão que, de acordo com nosso entendimento, deveria guiar a reflexão acerca destas considerações, seria: será que os pais têm esta condição? Como transmitir bons hábitos alimentares aos filhos, se os pais não têm condições concretas para isso?

Nota-se, de uma forma geral, como dito anteriormente, uma forte tendência na literatura científica a associar a obesidade a uma questão comportamental, a uma questão de escolha do sujeito. Nesse sentido, além dos autores citados até então, Fagundes, Ribeiro e Naspitz, ${ }^{22}$ afirmam que o desenvolvimento de obesidade se dá por conta de hábitos e comportamentos de vida inadequados no que se refere ao plano alimentar e na prática de atividade física. Outros autores também pensam de forma semelhante, como Afonso, Cunha e Oliveira ${ }^{2}$; Mendonça, Silva e Rivera ${ }^{23}$; Cattai, Rocha e Hintze ${ }^{26}$; Dias et al. ${ }^{28}$; Fonseca et al. ${ }^{39}$; Borges e César ${ }^{40}$; Fernandes et al. ${ }^{41}$ Estes últimos destacam que por muitas vezes, ocorre um comportamento inadequado do sujeito quanto ao controle de seu peso corporal. Estes autores não abordam, porém, o porquê deste "comportamento inadequado", ou melhor, o que determina esta prática.

Frente a essas abordagens superficiais, imediatas e naturalizadoras, é relevante atentar-nos para alguns dados referentes aos hábitos alimentares e prática de atividade física.

Dados apresentados pela Rede Interagencial de Informações para a Saúde, ${ }^{42}$ indicaram que no Brasil, no ano de 2008, 56.263.735 pessoas sobreviviam com menos de meio salário mínimo mensal, cerca de $\mathrm{R}$ \$255,00, havendo uma predominância de residentes na região norte. Nesse sentido, ainda de acordo com dados fornecidos pela RIPSA, ${ }^{43}$ a participação diária per capita das calorias de frutas, verduras e legumes no total de calorias da dieta da população da região norte do Brasil, era de 1,7\%, em contrapartida, na região sul, a participação era de 2,7\% no total de calorias consumidas, o que ainda é pouco. A análise destes dados evidencia, então, a existência de uma forte relação entre a renda mensal e o consumo de frutas, verduras e legumes, consumo este, negativamente relacionado ao ganho de massa adiposa. 
Podemos relacionar também a renda mensal da população brasileira com a prática de atividades físicas. Atualmente, o Brasil possui mais de 7 mil academias regularizadas, porém, apenas 2\% dos brasileiros vão a este tipo de estabelecimento. ${ }^{44}$

Quanto à prática de atividades físicas fora destes estabelecimentos, devemos considerar a influência dos altos índices de violência urbana em nosso país. De acordo com dados do Instituto Brasileiro de Geografia e Estatística, ${ }^{45}$ agressões e acidentes urbanos respondem por 12,5\% do total de mortes no Brasil e, em 15 Estados, estes fatores são mais letais do que o câncer.

Com isso, podemos compreender que os hábitos alimentares, bem como a prática de atividades físicas são orientados e determinados por fatores que transcendem ao comportamento individual e à vontade dos sujeitos, ou seja, estão relacionados às condições de vida concretas e materiais, impostas pelo nosso modo de organização social, capitalista.

\section{SOBRE OS FATORES GENÉTICOS, FISIOLÓGICOS E HEREDITÁRIOS COMO CAUSADORES DA OBESIDADE}

Grande ênfase se dá também aos fatores de ordem genética no desenvolvimento da obesidade. ${ }^{6,29,46}$ Alguns autores se referem à questão genética da obesidade como "herança familiar"'. Indica-se que a correlação entre sobrepeso dos pais e de filhos é grande e decorre do compartilhamento da hereditariedade e a do meio-ambiente. ${ }^{6}$

Ainda sobre a influência genética no desenvolvimento da obesidade, sabe-se que os fatores hormonais e neurais, que influenciam os sinais de curto e longo prazo relacionados à saciedade e à regulação do peso corporal normal, são determinados geneticamente. Defeitos na expressão e na interação desses fatores podem contribuir para o aumento do peso corporal. Há evidências, também, que fatores genéticos podem influenciar o gasto energético, principalmente, a taxa metabólica basal (TMB). Alguns estudos têm mostrado a ação do hormônio leptina no desenvolvimento da obesidade, visto que a leptina atua na redução do consumo alimentar e no aumento do gasto energético. Apesar de pessoas obesas apresentarem níveis elevados de leptina, a falha pode estar em seu receptor ou ocorrer por diminuição na sensibilidade do organismo aos efeitos da leptina. ${ }^{16}$ 
Outras desordens endócrinas como o hipotireoidismo e problemas no hipotálamo, alterações no metabolismo de corticosteróides, hipogonadismo em homens e ovariectomia em mulheres, síndrome de Cushing e síndrome dos ovários policísticos podem ainda conduzir à obesidade. ${ }^{47}$

Os aspectos genéticos, fisiológicos e hereditários abordados aqui são apenas uma pequena parcela da variedade desses aspectos que podem estar relacionados com a etiologia da obesidade e que merecem atenção dos pesquisadores para que haja a elucidação dos vários mecanismos pelos quais eles influenciam no desenvolvimento dessa patologia.

Uma crítica se faz, porém, na limitação à análise desses aspectos no processo de desenvolvimento da obesidade, deixando, por vezes, à margem, aspectos determinantes de natureza social, referentes à dinâmica e funcionamento da sociedade capitalista.

Sobre essa realidade, mais evidente nos dias atuais, faz-se importante considerarmos os escritos de Leontiev. ${ }^{48}$ Esse autor afirma que o desenvolvimento humano fez e faz com que cada vez mais as leis sócio-históricas interfiram nas mudanças fisiológicas do homem, ao invés das leis biológicas. Assim, a partir da hominização, cada vez mais, o que age sobre o homem são suas condições sóciohistóricas. Portanto, o que provoca as diferenças entre os homens, como, por exemplo, a condição corporal obesa e não obesa, são as desigualdades sociais provocadas pelas sociedades de classes, uma vez que os homens, diferentemente dos animais, não são biologicamente determinados. Isto não significa que não somos seres biológicos, mas que as relações sociais onde estamos inseridos interferem de forma decisiva na nossa condição biológica. Sobre essa questão, podemos destacar o seguinte trecho escrito por Marx e Engels: ${ }^{7}$

[...] a sociedade burguesa, por ser baseada numa forma de exploração do homem pelo homem que mistifica as relações sociais, também oculta a sua verdadeira natureza. Ao transformar as relações sociais em relações entre coisas, faz com que essas relações apareçam como se fossem naturais (p. 9-10).

É certo que os seres humanos nascem com certa predisposição genética pra desenvolver certas doenças. É importante considerarmos, no entanto, que o que vai determinar o desenvolvimento ou não destas doenças, são os determinantes sociais, materiais e concretos, inclusive no que diz respeito a ter ou não acesso aos melhores tratamentos e às melhores formas de prevenção de doenças. 


\section{SOBRE OS FATORES SOCIOECONÔMICOS E DEMOGRÁFICOS COMO CAUSADORES DA OBESIDADE}

Alguns autores salientam os fatores sócio-econômicos como determinantes para essa problemática. $^{23,46,49,50,51}$ Cavalcanti e Melo, ${ }^{50}$ destacam que o padrão alimentar vem sendo modificado ao longo da história em função de vários aspectos, como por exemplo, o maior poder aquisitivo da sociedade moderna, que permite ampliação do mercado consumidor e diversidade dos produtos nas prateleiras. Já para Ferreira e Magalhães, ${ }^{52}$ a obesidade emerge como mais uma face das desigualdades sociais no Brasil, assim como a desnutrição e as anemias carenciais. Para Gouveia e colaboradores, ${ }^{46}$ os fatores sócio-econômicos são os principais determinantes do desenvolvimento de obesidade, pois, tem estreita ligação com a dieta e a atividade física.

De acordo com Campos, Leite e Almeida, ${ }^{15}$ em países em desenvolvimento, como o Brasil, a obesidade está associada ao maior nível socioeconômico. Isso porque existe uma maior disponibilidade de alimentos com maior densidade energética e pela menor atividade física nesses estratos sociais. Em um estudo realizado com 1158 adolescentes, estes autores identificaram que a prevalência de sobrepeso e obesidade, em adolescentes do município de Fortaleza, ocorre principalmente nas classes sociais mais favorecidas.

Estudos de Zamai et al. ${ }^{53}$; Zamai e Moraes $^{54}$ analisaram o conhecimento de escolares de diversas escolas públicas em 20 municípios do Estado de São Paulo sobre as temáticas sobrepeso, obesidade e as doenças crônicas não transmissíveis e enfatizam que a alimentação incontrolada e falta de conhecimento são fatores agravante entre escolares do ensino fundamental e médio de diversos municípios, pois dentre os alunos do ensino fundamental 31,9\% apresentaram índices abaixo do peso; $10 \%$ peso normal; $39 \%$ apresentaram sobrepeso e 20,1\% receberam classificações de obesidade I. No ensino médio $15 \%$ apresentaram índices abaixo do peso; $16,5 \%$ peso normal; $31 \%$ sobrepeso e $38,5 \%$ receberam classificações de obesidade I.

Outro estudo mostra que, no período de 1989 a 2003, os homens continuaram apresentando aumento da obesidade independentemente de sua faixa de renda, enquanto, entre as mulheres, somente aquelas com baixa escolaridade e situadas nas faixas de renda mais baixas (entre as $40 \%$ mais pobres) apresentaram uma maior prevalência de obesidade. ${ }^{37}$ 
Considera-se que a comparação entre os estudos podem apresentar restrições, visto que os procedimentos para a categorização de níveis socioeconômicos são diversificados. ${ }^{15}$ Ainda de acordo com os autores, nos países desenvolvidos, a obesidade tende a ser mais freqüente nas camadas da população com menor renda,ocorrendo o inverso nos países em desenvolvimento.

As diferenças geográficas nos países expressam diferenciações na distribuição da obesidade. Inicialmente, verificou-se maior prevalência de excesso de gordura corporal nas regiões mais desenvolvidas (Sul, Sudeste e Centro-Oeste) do país e nos estratos de renda mais elevados, mas já se observa tendência de aumento da obesidade nas regiões Norte e Nordeste nos estratos de renda mais baixos. Assim, a comparação dos resultados dos estudos referidos acima nas regiões Norte e Nordeste com os das regiões Sul, Sudeste e Centro-Oeste permite assinalar a maior prevalência da obesidade nas últimas regiões citadas. Além disso, os estudos indicaram que a ocorrência desse agravo praticamente triplicou entre homens e mulheres maiores de vinte anos na região Nordeste e somente entre os homens do Sudeste. ${ }^{47}$

Em síntese, a maioria dos estudos que apontam os fatores sócio-econômicos como determinantes para o desenvolvimento de obesidade, tentam estabelecer uma correlação positiva entre o desenvolvimento de obesidade e o nível sócio-econômico de um país ou certa região, algo que não foi alcançado até então.

\section{SOBRE OS FATORES PSICOLÓGICOS, ESTRESSE, FUMO E ÁLCOOL COMO CAUSADORES DA OBESIDADE}

Também se destacam na literatura os fatores de ordem psicológica e psíquica, sendo estes, na maioria das vezes, ligados a questões comportamentais que resultam, por fim, na falta de adaptação social do sujeito e, conseqüente, desenvolvimento de transtornos psicológicos. Dentre estes fatores psicológicos apontados como relacionados com o desenvolvimento de obesidade, podemos citar: a baixa auto-estima, a ansiedade e a depressão., ${ }^{6,20}$

A esse respeito, é importante tratarmos da questão do trabalho em nossa sociedade. De acordo com Netto e Braz, ${ }^{10}$ a divisão social do trabalho faz com que a própria ação do homem se torne para este um poder alienado e a ele oposto, que o subjuga, em vez de ser ele a dominá-la. Assim que o trabalho começa a ser distribuído, cada homem tem uma atividade determinada e exclusiva que lhe é imposta e a qual não pode sair se não quiser perder seus meios de subsistência. E essa fixação da 
atividade social, segundo os autores, "escapa ao nosso controle, contraria as nossas expectativas e aniquila os nossos cálculos".

E isso tem forte relação com o estresse, promotor de problemas de ordem psíquica e psicológica, como ansiedade e depressão, importantes desencadeadores da obesidade.

No trato dos fatores psicológicos e psíquicos da obesidade, também é fundamental apresentarmos os escritos de Navarro. ${ }^{28}$ A autora, ao analisar a relação entre o trabalho, saúde e tempo livre dos trabalhadores na sociedade capitalista, afirma que o contexto da precarização do trabalho propiciou o crescimento da incidência, entre os trabalhadores, de doenças como a depressão, a síndrome do pânico, o estresse, fumo, alcoolismo, dentre outros transtornos que apresentam comprovada relação com as precárias condições de trabalho. Ainda de acordo com Navarro: ${ }^{28}$

\begin{abstract}
A análise da equação trabalho/saúde/tempo livre nos dias atuais passa, pois, pela compreensão da lógica que rege a intensificação do trabalho na sociedade capitalista contemporânea. Lógica esta que desemprega, extingue empregos formais e cria toda sorte de trabalhos precários; lógica que produz tecnologias altamente sofisticadas que permitem o aumento nos ganhos de produtividade, diminuição do tempo necessário para a produção e, ao mesmo tempo, amplia a jornada de trabalho e intensifica a utilização de horas extras em prejuízo da saúde e do tempo livre das pessoas. (p. 56)
\end{abstract}

Como exemplo dos efeitos desta lógica que rege a intensificação do trabalho na sociedade capitalista, pode verificar que a taxa de desemprego no Brasil vem aumentando: enquanto no ano 2000 o Brasil tinha uma taxa de desemprego de 7.5\%, em 2008, o Brasil contava com uma taxa de desemprego de $7.9 \% .{ }^{45}$ De acordo com dados do IBGE, ${ }^{11}$ nosso país fechou o ano de 2009 com uma taxa de $8,1 \%$, marcando um progressivo crescimento no número de desempregados no Brasil.

Além desse quadro de precarização do trabalho e aumento do desemprego, não podemos deixar de destacar o papel da mídia no desenvolvimento da obesidade. Esta, de acordo com Wanderley e Ferreira ${ }^{47}$ impõe ideal de saúde e beleza, difunde conselhos dietéticos, estéticos, esportivos, eróticos e psicológicos, e ainda, impõe um padrão idealizado e homogêneo de beleza, pouco provável de ser alcançado. Tal realidade pode contribuir de forma significativa para os aspectos psicológicos e psíquicos envolvidos na gênese da obesidade.

\title{
CONSIDERAÇÕES FINAIS
}

Como considerações finais, destacamos o atual quadro de superficialidade com que a obesidade vem sendo tratada na literatura, como se seu desenvolvimento fosse algo natural na sociedade e 
dependesse, principalmente, da vontade e comportamento do sujeito em relação à dieta e prática de atividade física. Com a apresentação de alguns dados, buscamos neste trabalho, indicar que os fatores causadores de obesidade estão enraizados na estrutura e funcionamento da sociedade capitalista, sistema de organização social onde o lucro é o objetivo final do trabalho humano. Nesse sentido, é evidente que enquanto estivermos vivendo nesse modo de organização social, não nos desenvolveremos plenamente e os problemas sociais como a obesidade não será sanada.

Identificamos, por isso, a necessidade de desenvolvimento de novas pesquisas sobre o tema, que entendam como fundamental o estabelecimento de uma relação direta entre os aspectos sócioeconômicos e estruturais da sociedade capitalista e o desenvolvimento da obesidade.

Assim, apontamos para a necessidade de uma transformação radical da sociedade capitalista, com vistas a uma organização social emancipada, onde não houvesse a divisão da sociedade em classes, onde trabalhar-se-ia muito pouco e dedicar-se-ia mais aos aspectos referentes ao desenvolvimento das potencialidades do ser humano e, consequentemente, com as questões relacionadas à saúde.

Enquanto não é instaurada uma nova forma de organização social, no entanto, pautada no ser humano e não no lucro, precisamos traçar novas estratégias e políticas sociais para amenizar o aumento de casos de obesidade no mundo.

\section{REFERÊNCIAS}

${ }^{1}$ CUNHA, A. C. P. T.; NETO, C. S. P. Indicadores de obesidade e estilo de vida de dois grupos de mulheres submetidas à cirurgia bariátrica. Fitness \& Performance Journal, Rio de Janeiro, v. 5, n. 3, p. 146-154, 2006.

${ }^{2}$ AFONSO, C. T.; CUNHA, C. F.; OLIVEIRA, T. R. P. R. Tratamento da obesidade na infância e adolescência: um a revisão da literatura. Revista Medica de Minas Gerais, Belo Horizonte, v. 18, n. 4, p. 131-138, 2008.

${ }^{3}$ ENES, C. C.; SLATER, B. Obesidade na adolescência e seus principais fatores determinantes. Revista Brasileira de Epidemiologia, São Paulo, v. 13, n. 1, p. 163-171, 2010.

${ }^{4}$ FERREIRA, J. S.; AYDOS, R. D. Prevalência de hipertensão arterial em crianças e adolescentes obesos. Ciência \& Saúde Coletiva, Rio de Janeiro, v. 15, n. 1, p. 97-104, 2010.

Conexões: revista da Faculdade de Educação Física da UNICAMP, Campinas, v. 10, n. 1, p. 121-141, jan./abr. 2012. 
${ }^{5}$ BARBIERI, A. F. Obesidade na adolescência: aspectos de adesão e permanência em programa de tratamento multiprofissional pautado na terapia comportamental. Revista Digital Efdeportes, Buenos Aires, ano 15, n. 143, abr. 010. Disponível em: http://www.efdeportes.com/efd 143/obesidade-na-adolescencia-aspectos-de-adesao-e-permanencia.htm. Acesso em: 13 maio 2010.

${ }^{6}$ DAlCAStAgnÉ, G. et al. A influência dos pais no estilodevidadosfilhosesuarelaçãocomaobesidade infantil. Revista Brasileira de Obesidade, Nutrição e Emagrecimento, São Paulo, v. 2, n. 7, p. 44-52, jan./fev. 2008.

${ }^{7}$ MARX, K.; ENGELS, F. A ideologia alemã. São Paulo: Expressão Popular, 2009.

${ }^{8}$ MARX, K.; ENGELS, F. Textos sobre educação e ensino. 2. ed. São Paulo: MORAES, 1992.

${ }^{9}$ NETTO, J. P. Relendo a Teoria Marxista da História. In: SAVIANI, D.; LOMBARDI, J. C.; SANFELICE, J. L. História e história da educação. Campinas: Autores Associados, p. 50-64, 1998.

${ }^{10}$ NETTO, J. P.; BRAZ, M. Economia política: uma introdução crítica. São Paulo: Cortez, v. 1, p. 71-122, 2006.

${ }^{11}$ NSTITUTO BRASILEIRO DE GEOGRAFIA E ESTATÍSTICA (IBGE). Distribuição das despesas monetária e não monetária média mensal familiar (\%) total classes de rendimento total e variação patrimonial mensal familiar (R\$) (1) classes de rendimento total e variação patrimonial mensal familiar, segundo os tipos de despesa. 2009. Disponível em: < http://www.ibge.gov.br/home/estatistica/populacao/condicaodevida/pof/2008_2009/tabelas_pdf/tab ela1_1_2.pdf> Acesso em: 14 nov. 2010.

${ }^{12}$ LEFÈVRE, F. O medicamento como mercadoria simbólica. São Paulo: Cortez, 1991.

${ }^{13}$ GONÇALVES, A. (Org.). Saúde coletiva e urgência em Educação Física e esportes. Campinas: Papirus, 1997. 
${ }^{14}$ LIBERATORE JÚNIOR, R. D. R.; SANTOS, L. R. Crianças e adolescentes obesos: estamos capacitados para atendê-los? Revista Paulista de Pediatria, São Paulo, v. 24, n. 1, p. 52-56, 2006.

${ }^{15}$ CAMPOS, L. A.; LEITE, A. J. M.; ALMEIDA, P. C. Nível socioeconômico e sua influência sobre a prevalência de sobrepeso e obesidade em escolares adolescentes do município de Fortaleza. Revista de Nutrição, Campinas, v. 19, n. 5, p. 531-538, set./out., 2006.

${ }^{16}$ FERREIRA, S. et al. Aspectos etiológicos e o papel do exercício físico na prevenção e controle da obesidade. Revista de Educação Física, São Paulo, n. 133, p. 15-24, mar. 2006.

${ }^{17}$ LOTTENBERG, A. M. P. Tratamento dietético da obesidade. Einstein, supl.1, p. 23-28, 2006.

${ }^{18}$ NOBRE, M. R. C.; DOMINGUES, R. Z. L.; SILVA, A. R.; et al. Prevalências de Sobrepeso, Obesidade e Hábitos de vida associados ao risco cardiovascular em alunos do ensino fundamental. Revista da Associação Médica Brasileira, São Paulo, v. 52, n. 2, p. 118-124, 2006.

${ }^{19}$ FILARDO, R. D.; PETROSKI, E. L. Prevalência de sobrepeso e obesidade em homens adultos segundo dois critérios de diagnóstico antropométrico. Motricidade, Santa Maria da Feira, v. 3, n. 4, p. 46-54, 2007.

${ }^{20}$ KEHER, G. M. et al. Prevenção e tratamento da obesidade: indicativos do sul do Brasil.Ciência, Cuidado \& Saúde, Maringá, v. 6, supl. 2), p. 427-432, 2007.

${ }^{21}$ LIMA, L. P.; SAMPAIO, H. A. C. Caracterização socioeconômica, antropométrica e alimentar de obesos graves. Ciência \& Saúde Coletiva, v. 12, n. 4, p. 1011-1020, 2007.

${ }^{22}$ FAGUNDES, A. L. N.; RIBEIRO, D. C.; NASPITZ, L. Prevalência de sobrepeso e obesidade em escolares da região de Parelheiros do município de São Paulo. Revista Paulista de Pediatria, São Paulo, v. 26, n. 3, p. 212-217, 2008. 
${ }^{23}$ FERRARI, H. G.; BARBOSA, M. H. Prevalência de sobrepeso e obesidade em escolares do ensino fundamental de escolas públicas de Limeira, São Paulo Conexões: revista da Faculdade de Educação Física da UNICAMP, Campinas, v. 6, nº especial, 2008

${ }^{24}$ BAPTISTA, M. N.; VARGAS, J. F.; BAPTISTA, A. S. D. Depressão e qualidade de vida em uma amostra brasileira de obesos mórbidos. Avaliação Psicológica, São Paulo, v. 7, n. 2, p. 235-247, 2008.

${ }^{25}$ CATTAI, G. B. P. et al. Programa de tratamento multiprofissional da obesidade: os desafios da prática. Ciencia,Cuidado \& Saude, Maringá, v. 7, p. 121-126, 2008.

${ }^{26}$ CHAVES, M. G. A. M. et al. Estudo da relação entre a alimentação escolar e a obesidade. HU Revista, Juiz de Fora, v. 34, n. 3, p. 191-197, jul./set. 2008.

${ }^{27}$ DIAS, L. C. G. D. et al. Sobrepeso e obesidade em crianças pré-escolares matriculadas em cinco centros de educação infantil de Botucatu, São Paulo. Revista Ciência em Extensão, v. 4, n. 1, p.107, 2008.

${ }^{28}$ NAVARRO, V. L. Trabalho, saúde e tempo livre sob os domínios do capital. In: PADILHA, V.

Dialética do lazer. São Paulo: Cortez, 2006. p. 50-76.

${ }^{29}$ COSTA, F. S. et al. Considerações acerca da avaliação psicológica das comorbidades psiquiátrica em obesos. Psicologia em Estudo, Maringá, v. 14, n. 2, p. 287-293, abr./jun. 2009.

${ }^{30}$ KUNKEL, N.; OLIVEIRA, W. F.; PERES, M. A. Excesso de peso e qualidade de vida relacionada à saúde em adolescentes de Florianópolis, SC. Revista de Saúde Pública, v. 43, n. 2, p. 226-35, 2009.

${ }^{31}$ RIBAS, S. A.; SILVA, L. C. S. Dislipidemia em Escolares na Rede Privada de Belém. Arquivos Brasileiros de Cardiologia, v. 92, n. 6, p. 446-451, 2009.

${ }^{32}$ CAMILO, D. F. et al. Obesity and asthma: association or coincidence? Jornal de Pediatria, Rio de Janeiro, v. 86, n. 1, 2010.

Conexões: revista da Faculdade de Educação Física da UNICAMP, Campinas, v. 10, n. 1, p. 121-141, jan./abr. 2012. ISSN: 1983-9030 
${ }^{33}$ MENDONÇA, M. R. T.; SILVA, M. A. M.; RIVERA, I. R.; et al. Prevalência de sobrepeso e obesidade em crianças e adolescentes da cidade de Maceió. Revista da Associação Médica Brasileira, São Paulo, v. 56, n. 2, p. 192-196, 2010.

${ }^{34}$ FERREIRA, A. B. H. Novo Dicionário da língua portuguesa. 2. ed. Rio de Janeiro: Nova Fronteira, 1986.

${ }^{35}$ CAVAlCANTI, A. P. R.; DIAS, M. R.; COSTA, M. J. C. Psicologia e nutrição: predizendo a intenção comportamental de aderir a dietas de redução de peso entre obesos de baixa renda. Estudos de Psicologia, Natal, v. 10, n. 1, p. 121-129, 2005.

${ }^{36}$ SOUZA, S. Obesidade e consumo de alimentos no Brasil: uma análise das pesquisas de orçamentos familiares do IBGE, 2009. Disponível em: <http://www.slideshare.net/ regulacaoalimentos/obesidade-e-consumo-de-alimentos-no-brasil-uma-anlise-das-pesquisas-deoramentos-familiares-do-ibge> Acesso em: 19 fev. 2011.

${ }^{37}$ LOPES, P. C. S.; PRADO, S. R. L. A.; COLOMBO, P. Fatores de risco associados à obesidade e sobrepeso em crianças em idade escolar. Revista Brasileira de Enfermagem, Brasília, v. 63, n. 1, p. 73-78, jan./fev. 2010.

${ }^{38}$ RODRIGUES, E. M.; BOOG, M. C. F. Problematização como estratégia de educação nutricional com adolescentes obesos. Caderno de Saúde Pública, Rio de Janeiro, v. 22, n. 5, p. 923-931, mai., 2006.

${ }^{39}$ FONSECA, H. et al. Obesidade na adolescência: uma proposta de intervenção. Acta Pediatrica Portuguesa, v. 39, n. 2, p. 53-56, 2008.

${ }^{40}$ BORGES, R. G.; CÉSAR, T. B. Aplicação de dois critérios antropométricos na avaliação da prevalência de sobrepeso e obesidade em pré-escolares. Alimentos e Nutrição, Araraquara, v.16, n.4, p. 337-342, out./dez. 2005.

${ }^{41}$ FERNANDES, R. A. et al. Prevalência de Sobrepeso e Obesidade em alunos de escolas privadas do município de Presidente Prudente. Revista Brasileira de Cineantropometria e Desempenho Humano, Florianópolis, v. 9, n. 1, p. 21-27, 2007.

Conexões: revista da Faculdade de Educação Física da UNICAMP, Campinas, v. 10, n. 1, p. 121-141, jan./abr. 2012. ISSN: 1983-9030 
${ }^{42}$ REDE INTERAGENCIAL DE INFORMAÇÕES PARA A SAÚDE. (RIPSA). Proporção de pessoas com baixa renda $(\mathbf{1 / 2} \mathbf{~ s m})$. 2008. Disponível em: <http://tabnet.datasus.gov. br/cgi/tabcgi.exe?idb2009/b05.def>. Acesso em: 13 nov. 2010.

${ }^{43}$ REDE INTERAGENCIAL DE INFORMAÇÕES PARA A SAÚDE (RIPSA). Participação diária per capita das calorias de frutas, verduras e legumes no total de calorias da dieta. 2002. Disponível em: <http://tabnet.datasus.gov.br/cgi/idb2009/g12.htm〉. Acesso em: 14 nov. 2010.

${ }^{44}$ AVELAR, M. Academia: um negócio muito além da forma física. 2009. Disponível em: < http://www.educacaofisica.com.br/noticias_mostrar.asp?id=6235>. Acesso em: 14 nov. 2010.

${ }^{45}$ NSTITUTO BRASILEIRO DE GEOGRAFIA E ESTATÍSTICA (IBGE). Taxa de mortalidade por causas externas. 2008. Disponível em: < http://www.ibge.gov.br/home/ estatistica/populacao/condicaodevida/indicadoresminimos/defaulttab.shtm> Acesso em: 14 nov. 2010.

${ }^{46}$ GOUVEIA, E. R. et al. Atividade física, aptidão e sobrepeso em crianças e adolescentes: "o estudo de crescimento da madeira". Revista Brasileira de Educação Física e Esportes, São Paulo, v. 21, n. 2, p. 95-106, abr./jun. 2007.

${ }^{47}$ WANDERLEY, E. N.; FERREIRA, V. A. Obesidade: uma perspectiva plural. Ciência \& Saúde Coletiva, v. 15, n. 1, p. 185-194, 2010.

${ }^{48}$ LEONTIEV, A. O desenvolvimento do psiquismo. 2. ed. São Paulo: Centauro, 2004.

${ }^{49}$ FARIAS JÚNIOR, J. C.; SILVA, K. S. Sobrepeso/Obesidade em Adolescentes Escolares da Cidade de João Pessoa - PB: prevalência e associação com fatores demográficos e socioeconômicos. Revista Brasileira de Medicina do Esporte, v. 14, n. 2, mar./abr., 2008.

${ }^{50}$ CaVAlCANTI, A. C.; MELO, A. M. C. A. Mídia, comportamento alimentar e obesidade na infância e na adolescência: uma revisão. Revista Brasileira de Nutrição Clinica, São Paulo, v. 23, n. 3, p. 199-203, 2008. 
${ }^{51}$ GIGANTE, D. P.; MOURA, E. C.; SARDINHA, L. M. V. Prevalência de excesso de peso e obesidade e fatores associados, Brasil. Revista de Saúde Pública, v. 43, p. 83-89, 2006.

${ }^{52}$ FERREIRA, V. A.; MAGALHÃES, R. Obesidade no Brasil: tendências atuais. Revista Portuguesa de Saúde Pública, Lisboa, v. 24, n. 2, jul./dez. 2006.

${ }^{53}$ ZAMAI, C. A.; BANKOFF, A. D. P.; RODRIGUES, A.; SILVA, J. F. Conhecimento X Incidencia de fatores de risco entre escolares do ensino fundamental e médio do estado de São Paulo. Movimento e Percepção, Espírito Santo do Pinhal, v. 4, n. 4-5, 2004.

${ }^{54}$ ZAMAI, C. A.; MORAIS, J. C. Análise da incidência de sobrepeso e obesidade entre escolares de 7 a 10 anos de uma escola pública do distrito de Sousas - SP. Buenos Aires: Revista Efdeportes, año 13, n. 121, Jun/2008. Disponível em: http://www.efdeportes.com/ Acesso em: 11 mar. 2012. 